\title{
ACOUSTIC INSULATION PROVIDED BY A SINGLE WALL SEPARATING TWO CONTIGUOUS TUNNELS VIA BEM
}

\author{
P. Santos and A. Tadeu \\ Department of Civil Engineering, Faculty of Sciences and Technology, University of Coimbra, \\ 3030-290 Coimbra, Portugal.E-mail:pfsantos@dec.uc.pt
}

(Received 13 August 2001, and in final form 14 December 2001)

\begin{abstract}
This paper describes the computation of the acoustic insulation provided by a single simple wall separating two contiguous tunnels when a steady state, spatially sinusoidal, harmonic line load pressure excites one of the tunnels. The Boundary Element Method (BEM), formulated in the frequency domain, is used to compute the acoustic pressure in the two tunnels. The insulation conferred by the wall is characterized, identifying the location of insulation dips in the frequency domain with those dips related to its own natural dynamic vibration modes and those related to the natural vibration modes of the tunnels. This model is also used to assess how the dimension of the rooms, and the rigidity and thickness of the wall affect acoustic insulation. The transmission loss results obtained via the BEM are then compared with those provided by simplified analytical models such as the mass law.
\end{abstract}

(C) 2002 Published by Elsevier Science Ltd.

\section{INTRODUCTION}

The steadily growing impact of low-frequency indoor and outdoor noise sources such as due to music energy, aircraft noise and traffic noise $[1,2]$ motivated researchers to develop practical and theoretical interest on the sound transmission in buildings at low frequencies [3]. Low-frequency noise is indeed common at residential noise and has received less attention. Low-frequency noise differs from high-frequency noise since it suffers less attenuation due to walls and other structures, it travels great distances and loses little energy through atmospheric and ground attenuation. Ear-protection devices are also less effective against it [2].

Sound is transmitted through a partition wall by the vibration of the element with the mass and sound frequency being relevant variables. Airborne sound insulation improves with element mass increment, as a result of increasing forces of inertia. When the frequency of the incident sound is increased, for the same element mass, acoustic insulation rises owing to a fall in the element's vibration power and to greater dissipation of sound energy.

The acoustic insulation of a partition element is affected by variables other than mass and frequency. These include the existence of weak points in the insulation, the angle of incidence of the waves, the damping of the element, the rigidity and, in the case of multiple elements, the number of panels, their individual characteristics and how they are separated.

In a real situation, the transmission of sound between two contiguous rooms depends not only on the separation elements, but also on the connections between the surrounding 
elements, and on how propagation proceeds inside the emitting and receptor rooms. The vibration eigenmodes of the rooms excited determine how propagation occurs in this process.

Airborne wall sound insulation is a classic problem in acoustics. The first publications on it appeared at the beginning of the 20th century [4]. However, the development of theoretical simplified solutions to predict the sound insulation of building elements did not appear until the middle of the 20th century, when London developed theoretical equations to compute the transmission of sound through single and double walls [5-7]. Since then, the design of sound separating walls has been performed using classical analytical models.

The prediction of the sound insulation conferred by a single panel above, below, and in the vicinity of the coincidence effect has motivated different researchers to propose other simplified methods [8-11]. Callister et al. [12] used a combination of the prediction methods presented by Cremer [8], Sewell [9] and Sharp [10] to evaluate the sound transmission loss of a single-layer panel. Novikov [13] described the sound insulation of finite plates at low frequencies of excitation as the mass law plus a correction coefficient.

An infinite plate model, a baffled plane model and a room-plate-room model were used by Osipov et al. [14] to predict airborne sound transmission by single partitions at low frequencies. In these theoretical simplified models, the walls are assumed to be thin compared with the bending wavelength, and harmonic pure bending wave motion according to Kirchhoff's theory is adopted. Their results reveal that the sound insulation at low frequencies depends not only on the separating wall properties, but also on the geometry and dimensions of the contiguous rooms.

As the problem of sound insulation is quite complex, laboratory tests have been used to find the insulation of panels. The two-room method is the laboratory technique most often used to determine sound insulation [15-17]. The test facilities are built with a view to prevent flanking transmissions, in an effort to guarantee that the only path for the transmission of sound is via the test specimen [18]. However, the low-frequency range is not included in current standards on sound insulation measurements. Work by different authors [1-3, 19] have shown that the sound reduction index in this range depends on several parameters, e.g., the room's dimensions, the reverberation time, the position of sound source. Therefore, it is very difficult to extrapolate laboratory results to situations with different geometry and dimensions of the rooms or the partition.

It is not easy to measure the sound insulation provided by a partition construction element separating two compartments, for low frequencies (below $400 \mathrm{~Hz}$ ). The reproducibility of sound insulation measurements is not satisfactory at frequencies below $100 \mathrm{~Hz}$ for typical European transmission rooms with a volume of $50-70 \mathrm{~m}^{3}$ [20, 21]. It should be noted that no standard takes enough account of the fluctuating nature of noise, and fails to make a sufficient correction for large fluctuations [1].

Various numerical schemes for predicting the sound insulation of a separating wall have been proposed and developed. The Statistical Energy Analysis (SEA) method is particularly suitable for studying complex problems such as sound transmission [22]. This method was first introduced in the 1960s [23-25] and it brought together two distinct areas of study: room acoustics and the study of coupled modes. Before the advent of this method, the simultaneous study of a room's acoustic properties and its natural vibration modes was only practicable for the few first modes.

By this method, the acoustic system is divided into diverse subsystems. Each subsystem requires the knowledge of some properties to compute the global acoustical response, such as dimensions, density and wave speed. The global acoustic response is computed by the interaction of the different subsystems. One of the basic principles of this method is that only a minimum amount of information about a system is needed to compute the 
performance of an acoustic system. However, the SEA model is unreliable at low frequencies owing to the statistical uncertainties that occur when there are few resonant modes in each of the subsystems. The Finite Elements Model (FEM) is not affected by this limitation at low frequency. The SEA and the FEM were used by Steel and Craik [26] to compute the sound transmission between walls. The comparison of these results with measured data reveals that the FEM can be used for determining the coupling between subsystems. Hynnä et al. [27] used the SEA model to predict the structure-borne sound transmission in large welded ships' structures. They applied a pre-processing programme, frequently used in the context of the FEM, to reduce the modelling work. The SEA model has recently been used by Craik and Smith [28] to compute the sound transmission through double-leaf lightweight partitions. The wall is modelled as a single subsystem at low frequencies, while at higher frequencies the SEA model utilizes a number of interconnected subsystems.

Two different models were used by Kropp and Rebillard [29] to compute the sound insulation of double-wall constructions at low frequencies: a matrix formulation, which avoids the limitations in the thickness of the layers that occur with the Kirchhoff or Mindlin theories; a model which describes the double-wall construction as two bending plates connected by an elastic interlayer represented by uncoupled springs, which does not take into account the shear stiffness.

Other well-established numerical techniques, such as the finite element and finite difference methods, have not been used to compute sound insulation, given the high computation cost entailed. They have failed because the domain being analyzed has to be fully discretized, and very fine meshes are needed to solve excitations at high frequencies.

Osipov et al. [30] used the FEM to study the effect of room dimension on the sound insulation of a separating wall at low frequencies. The FEM was also used by Maluski and Gibbs [31] to predict the sound insulation between adjacent rooms at low frequency, and to compare the results with experimental data. Their results show that the sound insulation provided by a separating wall, at low frequencies, is strongly dependent on the modal characteristics of the sound field within each compartment.

The low-frequency diffuse field transmission loss through double-wall sound barriers with elastic porous linings was calculated by Sgard et al. [32]. They used a FEM to model the different layers of the sound barrier, coupled to a variational boundary element method to account for fluid loading. The diffuse field is assumed to be a combination of uncorrelated freely propagating plane waves with equal amplitude, no two of which are travelling in the same direction.

Most of the methods described are limited to laterally infinite sound panels, and the predictions at low frequencies are also limited. The diffuse field is the basis of standardized transmission loss measurements and, usually, these models assume plane wave excitation, which represents another limitation. Also, it is classically assumed in most of the abovecited references that the fluid loading is negligible.

The Boundary Element Method (BEM) is used in this work to compute the sound insulation provided by an elastic wall that separates two air-filled tunnels at low frequencies. In the simulated model used, a 3-D source is placed in one of the tunnels, while the response is computed inside the two tunnels at low frequencies. This method circumvents the limitations of the thickness of the layer, found with Kirchhoff or Mindlin theories; it models the surfaces of the tunnel and the separating wall completely, and fully takes into account the coupling between the fluid (air) and the solid formation.

The steady improvement in computer performance now means that numerical methods, such as the BEM, can be developed, and these are being applied more frequently. The BEM has already been used to simulate the propagation of waves between two fluid-filled 
boreholes, when a source is placed in one borehole, and the other hosts the receivers [33]. This models a seismic prospecting technique, known as cross-hole surveying, commonly used to determine the characteristics of the elastic medium between two boreholes.

This method has been used by Bouchon [34] to study wave propagation in an infinite open borehole in layered isotropic media. Dong et al. [35] subsequently extended this work by adding casing and cement to the formation, and assessing its effect, and by incorporating transversely isotropic layers. Their work used an indirect BEM to model source radiation from open and cased boreholes in layered transversely isotropic media.

The effect of irregular boreholes on the response has been studied by Randall [36] and Tadeu and Santos [33], simulating the deformation of the borehole to represent the mechanical action of the drill string in vertically deviated wells, rock failure adjacent to a drilled borehole, plastic deformation, or washing out of the borehole in soft or poorly consolidated rocks, as reported by Bell and Gough [37] and by Zheng et al. [38].

The BEM could be regarded as the best tool for modelling and analyzing wave propagation in an unbounded medium because it automatically satisfies the farfield conditions and only the boundary of the elements being studied need to be discretized.

The structure of the paper is as follows: first, a brief definition of the 3-D problem is given, and then the BEM is formulated in the frequency domain. The results are validated by applying them to the situation of a cylindrical, air-filled circular tunnel for which analytical solutions are known. The theoretical mass law is then described, and the importance of variables to the definition of acoustic insulation is explained. Such variables include the mass, frequency, coincidence effect and eigenmodes of an enclosed room. Finally, simulations are performed for different tunnel configurations. The computed insulation curves obtained are then compared with those predicted by the simplified analytical model. The influence of wall thickness, separating the tunnels, the tunnels geometry (height and width), the receivers' position, the mechanical properties of the elastic material and the apparent wave velocity are all analyzed.

\section{BEM FORMULATION}

Twin tunnels, separated by a concrete wall, are driven along the $z$ direction in a elastic medium (see Figure 1). The lining of the tunnel is assumed to be concrete, with density $\rho$, allowing a shear wave velocity of $\beta$ and a compressional wave velocity of $\alpha$. The material surrounding the lining is assumed to have similar properties as those of the lining. Since the aim of the present model is to calculate the airborne sound insulation conferred by a wall, separating two tunnels, this simplification is not significant because the amount of energy crossing the lining of the tunnel is very small compared with the energy that goes through the separating wall. The fluid (air) inside the tunnels has a density $\rho_{a}$ and permits a compressional wave velocity $\alpha_{a}$. A dilatational point source is placed in one of the tunnels at position $\left(x_{0}, y_{0}, z_{0}\right)$, oscillating with a frequency $\omega$. The pressure incident field is expressed by

$$
p_{\text {inc }}=\frac{A \mathrm{e}^{\mathrm{i} \omega / \alpha_{a}\left(\alpha t-\sqrt{\left(x-x_{0}\right)^{2}+\left(y-y_{0}\right)^{2}+\left(z-z_{0}\right)^{2}}\right)}}{\sqrt{\left(x-x_{0}\right)^{2}+\left(y-y_{0}\right)^{2}+\left(z-z_{0}\right)^{2}}}
$$

in which $A$ is the wave amplitude and $\mathrm{i}=\sqrt{-1}$.

As the geometry of the tunnels does not change along the $z$ direction, the solution can be obtained as a summation of 2-D problems for varying effective 


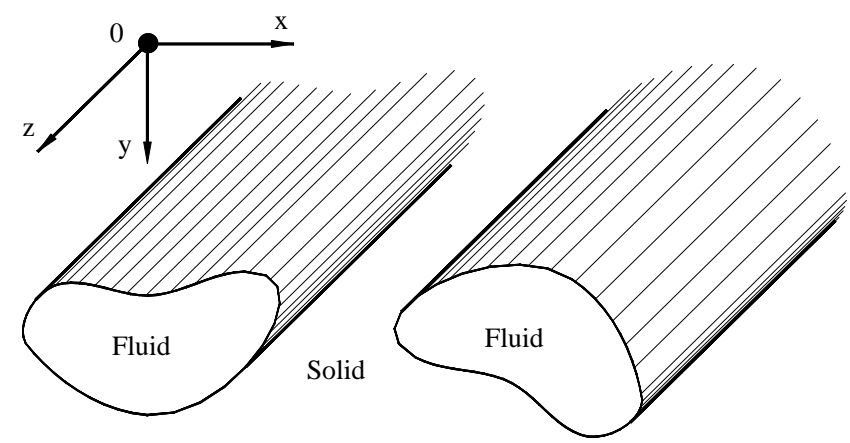

Figure 1. Geometry of the problem.

wavenumbers [39],

$$
k_{\alpha_{a}}=\sqrt{\frac{\omega^{2}}{\alpha_{a}^{2}}-k_{z}^{2}}, \quad \operatorname{Im} k_{\alpha_{a}}<0
$$

where $k_{z}$ is the axial wavenumber after Fourier transformation of the problem in the $z$ direction. The incident field in this frequency wavenumber domain is given by

$$
\hat{p}_{\text {inc }}\left(\omega, x, y, k_{z}\right)=\frac{-\mathrm{i} A}{2} \mathrm{H}_{0}^{(2)}\left(k_{\alpha_{a}} \sqrt{\left(x-x_{0}\right)^{2}+\left(y-y_{0}\right)^{2}}\right)
$$

in which the $\mathrm{H}_{n}^{(2)}(\ldots)$ are Hankel functions of the second kind of order $n$. This is often referred to in the literature as a $2 \frac{1}{2}$-D problem, because the geometry is $2 \frac{1}{2}$-D and the source is 3-D.

As the lining is assumed to be surrounded by material with identical properties, the BEM only requires the discretization of the inner surfaces of the tunnels. The BEM equations that are applied to this problem have been applied by the authors to the solution of the wave propagation in a fluid-filled borehole [33]. The system of equations required for the solution is arranged so as to impose the continuity of the normal displacements and normal stresses, and null shear stresses along the boundary of the fluid-filled boreholes. This system of equations requires the evaluation of the following integrals along the appropriately discretized boundary of the borehole,

$$
\begin{gathered}
H_{i j}^{(s) k l}=\int_{C_{l}} H_{i j}^{(s)}\left(x_{k}, x_{l}, n_{l}\right) \mathrm{d} C_{l} \quad(i, j=1,2,3), \\
H_{a_{1}}^{(a) k l}=\int_{C_{l}} H_{a_{1}}^{(s)}\left(x_{k}, x_{l}, n_{l}\right) \mathrm{d} C_{l}, \\
G_{i j}^{(s) k l}=\int_{C_{l}} G_{i j}^{(s)}\left(x_{k}, x_{l}\right) \mathrm{d} C_{l} \quad(i=1,2,3 ; j=1) \\
G_{a_{1}}^{(a) k l}=\int_{C_{l}} G_{a_{1}}^{(a)}\left(x_{k}, x_{l}\right) \mathrm{d} C_{l}
\end{gathered}
$$

in which $H_{i j}^{(s)}\left(x_{k}, x_{l}, n_{l}\right)$ and $G_{i j}^{(s)}\left(x_{k}, x_{l}\right)$ are, respectively, the Green's tensor for traction and displacement components in the elastic medium, at point $x_{l}$ in the direction $j$ caused by a concentrated load acting at the source point $x_{k}$ in the direction $i$; $H_{a_{1}}^{(a)}\left(x_{k}, x_{l}, n_{l}\right)$ are the components of the Green's tensor for pressure in the fluid medium, at point $x_{l}$ caused by a pressure load acting at the source point $x_{k} ; G_{a_{1}}^{(a)}\left(x_{k}, x_{l}\right)$ are the components of the 
Green's tensor for displacement in the fluid medium, at point $x_{l}$ in the normal direction, caused by a pressure load acting at the source point $x_{k} ; n_{l}$ is the unit outward normal for the $l$ th boundary segment $C_{i}$; the subscripts $i, j=1,2,3$ denote the normal, tangential and $z$ directions respectively. These equations are conveniently transformed from the $x, y, z$ Cartesian co-ordinate system by means of standard vector transformation operators. The required $2 \frac{1}{2}-\mathrm{D}$ fundamental solution (the Green functions) and stress functions in Cartesian co-ordinates, for the elastic and fluid media, are listed in Appendix A.

The required integrations in equation (4) are performed analytically for the loaded element $[40,41]$. A Gaussian quadrature scheme is used when the element to be integrated is not the loaded element.

\section{BEM VALIDATION}

The BEM algorithm was implemented and validated by applying it to a cylindrical circular air-filled tunnel for which the solution is known in closed form [42]. The mechanical properties of the elastic medium (concrete) and the fluid medium of the tunnel (air) are listed in Figure 2.

The fluid-filled circular tunnel is subjected to a point dilatational load applied at point $O$, as represented in Figure 2. The response is computed along a plane, placed perpendicular to the longitudinal axis of the tunnel, over a fine grid of receivers, as illustrated in Figure 3(a). The scattered pressure field inside the borehole and the vertical displacement field in the solid formation, when a harmonic pressure load of $600 \mathrm{~Hz}$ is excited at $k_{z}=0.5 \mathrm{rad} / \mathrm{m}$, are represented in Figure 3(b).

All graphics displayed have been normalized by dividing the response by the maximum displacement and pressure, both outside and inside the borehole. The BEM results were computed with a different number of boundary elements. Figures 3(c) and 3(d) displays the BEM error obtained by the difference with the analytical results.

The BEM accuracy improves as smaller boundary elements are used to model the response, as expected. In these examples, the ratio between the wavelength of the compressional sound waves and the length of boundary elements increases from 8 (21

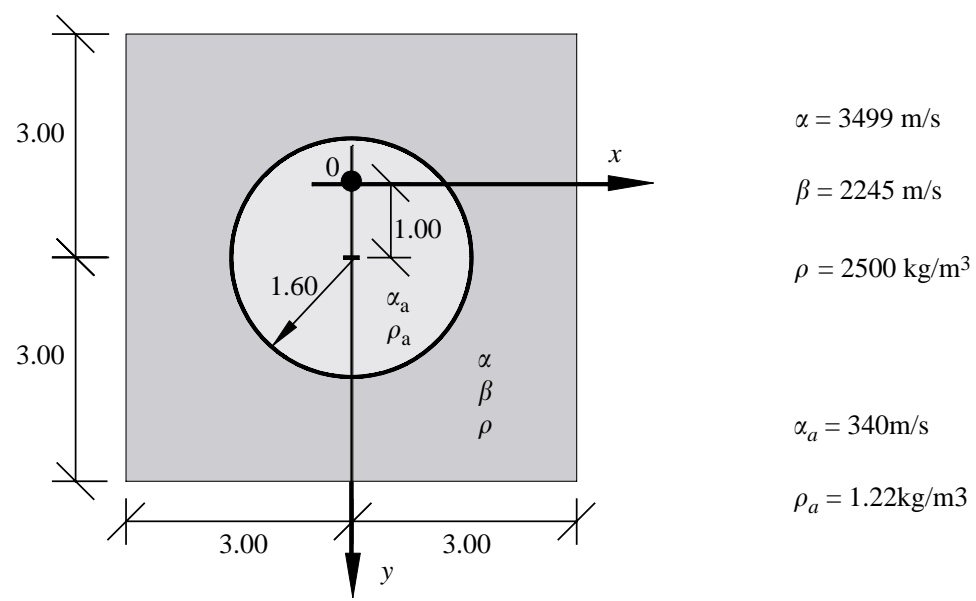

Figure 2. Circular cylindrical inclusion in an unbounded elastic medium. 


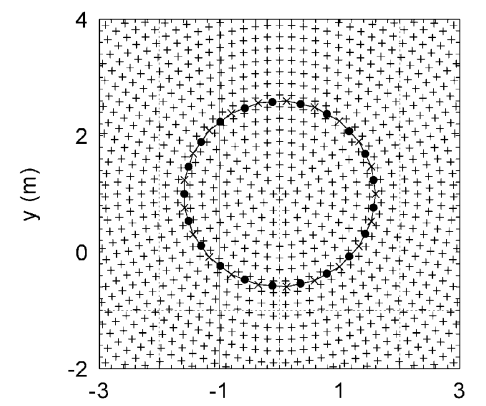

(a)

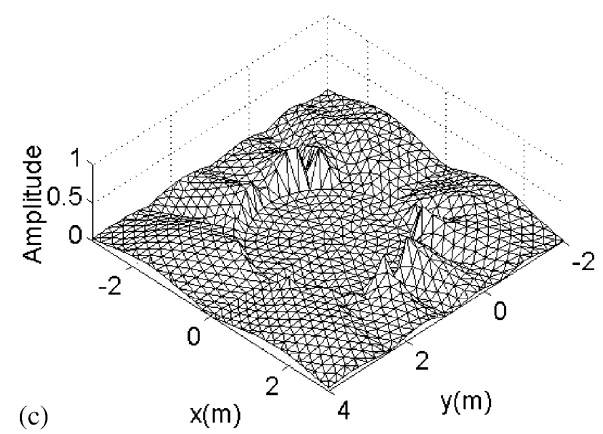

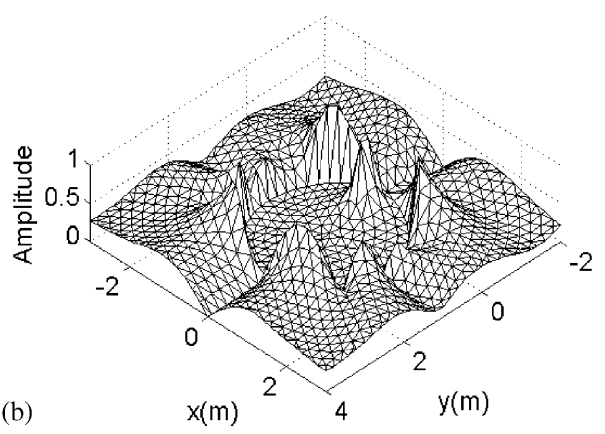

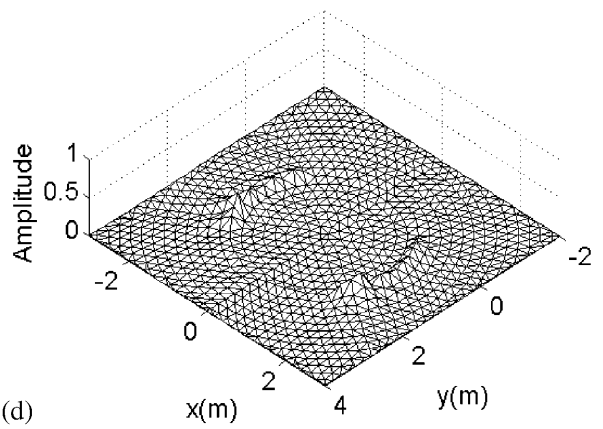

Figure 3. Validation of the BEM: (a) typical BEM mesh; (b) analytical response; (c) normalized BEM error (21 elements); (d) normalized BEM error (43 elements).

boundary elements) to 16 (43 boundary elements). The results also show that the BEM solution improves as the distance to the surface of the inclusion increases.

\section{ACOUSTIC INSULATION}

\subsection{THEORETICAL MASS LAW}

One of the commonly used approximations for predicting the sound reduction index of a single panel wall is known as the Law of Theoretical Mass, or the Law of Theoretical Frequency [11],

$$
R=10 \log \left[1+\left(\frac{\pi f M \cos \theta}{\rho_{a} \alpha_{a}}\right)^{2}\right] \mathrm{dB},
$$

where $R$ denotes the sound reduction index, $f$ is the frequency $(\mathrm{Hz}), M$ is the mass per unit area of the panel $\left(\mathrm{kg} / \mathrm{m}^{2}\right)$ and $\theta$ is the angle of incidence.

This Theoretical Law adopts certain simplifications. It assumes that the partition element is infinite, that it behaves like a group of juxtaposed masses with independent displacement, that the partition element has null damping forces and that the incidence waves are plane waves. This equation predicts an increase in the sound reduction index of about $6 \mathrm{~dB}$ for each doubling of the mass per unit area.

Real division panels have finite size and they are struck by an infinite number of plane waves with differing angles of incidence, causing a diffuse field. After some mathematical 
manipulations, assuming the lack of waves at grazing incidence, the mass law can be assumed as

$$
R=20 \log (M f)-47 \quad \mathrm{~dB} .
$$

This simplified method does not take into account the localized dips in sound insulation provided by a wall due to its dynamic behaviour (rigidity of the partition element and its damping).

\subsection{PROPAGATION OF BENDING WAVES}

If one assumes the existence of an infinite thin plate, where the fluid coupling is negligible, the bending waves can travel along the plate with a velocity $\left(c_{L}\right)$ given by [43]

$$
c_{L}=\left(\frac{D \omega^{2}}{\rho h}\right)^{0.25}
$$

where $h$ is the panel thickness, $\omega=2 \pi f, D=\left(h^{3} E\right) /\left[12\left(1-v^{2}\right)\right], E$ is Young's modulus $\left(\mathrm{N} / \mathrm{m}^{2}\right), v$ is the Poisson ratio and $\rho$ is the plate material density $\left(\mathrm{kg} / \mathrm{m}^{3}\right)$.

The incident sound waves strike the panel from many directions. When the wavelength of sound air projected on the wall equals the wavelength of the wall bending waves, the movement of the partition panel is assumed to increase, leading to a low sound insulation. This happens when

$$
\omega=\left(\frac{\alpha_{a}}{\sin \phi}\right)^{2} \sqrt{\frac{\rho h}{D}},
$$

where $\phi$ is the incidence angle of the sound, relative to the element perpendicular direction.

The critical frequency corresponds to the case when $\phi$ is equal to $90^{\circ}$, known as the coincidence effect. In this case, we have

$$
f_{c}=\frac{\alpha_{a}^{2}}{1.81 h} \sqrt{\frac{\rho\left(1-v^{2}\right)}{E}} \quad(\mathrm{~Hz}) .
$$

\subsection{TRANSVERSE VIBRATION OF THE PANEL IN PURE FLEXURE}

The transverse movement of the wall affects its capacity to transfer energy to the surrounding space and, therefore, its sound insulation, producing dips in insulation. These dips are mainly going to occur at eigenfrequencies related to the panel's flexure-induced transverse movement.

\subsection{DYNAMIC RESPONSE OF A PARALLELEPIPED ROOM}

The precise characterization of a sound field which is established in an enclosed space, in the presence of a sound source, requires a complex physical-mathematical treatment. It involves variables that are difficult to quantify, such as the characteristics of sound energy dissipation in the air and in the surrounding medium, and those of the sound sources [44-46].

The formation of stationary waves is one of the factors that most influences the vibration properties of an enclosed tunnel, which occurs at

$$
f_{q r}=\frac{\alpha_{a}}{2} \sqrt{\left(\frac{q}{a}\right)^{2}+\left(\frac{r}{b}\right)^{2}} \quad(\mathrm{~Hz}),
$$


where $a, b$ are the tunnel dimensions (height and width, respectively) and $q$ and $r$ are integers $(0,1,2, \ldots)$.

\section{NUMERICAL APPLICATIONS}

The geometry of the twin concrete rectangular tunnels, and the concrete separating wall, driven along the $z$ direction in elastic medium, is illustrated in Figure 4(a). As described above, the lining of the tunnel is assumed to be thick and to have material properties similar to those of the surrounding elastic medium. The tunnels' surfaces are modelled with a number of boundary elements that increases with the frequency excitation of the harmonic source. The ratio between the wavelength of the incident waves and the length of the boundary elements is kept to a minimum of 10 . The number of boundary elements used to model each tunnel is never less than 80 . Given the small distance between the two faces of the separating wall, the length of boundary elements modelling the wall is at least 8 times smaller than its thickness.

In our examples, the material properties of the concrete $(\alpha=3499 \mathrm{~m} / \mathrm{s}, \beta=2245 \mathrm{~m} / \mathrm{s}$, $\left.\rho=2500 \mathrm{~kg} / \mathrm{m}^{3}\right)$, and of the air filling the tunnel $\left(\rho_{a}=1.22 \mathrm{~kg} / \mathrm{m}^{3}, \alpha_{a}=340 \mathrm{~m} / \mathrm{s}\right)$, are kept constant.

The computations were performed for two different positions of the source: in the centre of the tunnel (source 1), and in the close vicinity of the separating wall (source 2), as in Figure 4(a). Given the symmetry of the problem, the BEM computations use this characteristic of the geometry to improve its efficiency.

The response was calculated for pairs of receivers when analyzing the response at particular positions, while a grid of receivers was used to compute the average sound insulation provided by the wall separating the two tunnels (see Figure 4(b)).

The full 3-D results require high computational effort given the large number of 2-D solutions that needed to be integrated, thereby making the full integration far too time consuming. Next, simulations are performed following waves with different spatial sinusoidal variation along the $z$ direction, that is, with different apparent wave velocities along the $z$-axis, to quantitatively study the 3-D effects of the acoustic insulation. This apparent wave velocity $(c)$ results from waves arriving at the $z$-axis with a path inclination given by $\arccos \left(\alpha_{a} / c\right)$, where $\alpha_{a}$ is the true wave velocity (see Figure 5). In the equations presented above $k_{z}$ is taken to be $\omega / c$. Waves arriving at the receivers with a $90^{\circ}$

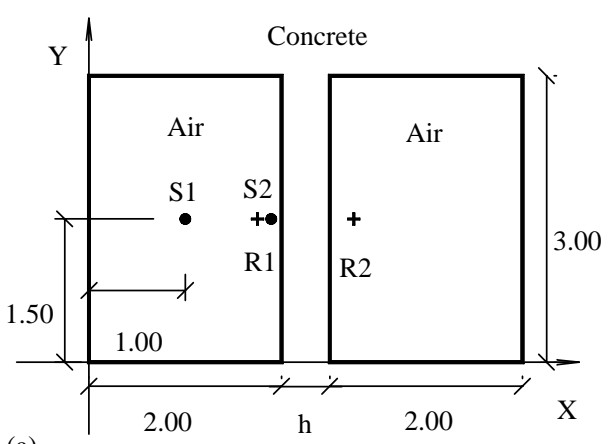

(a)

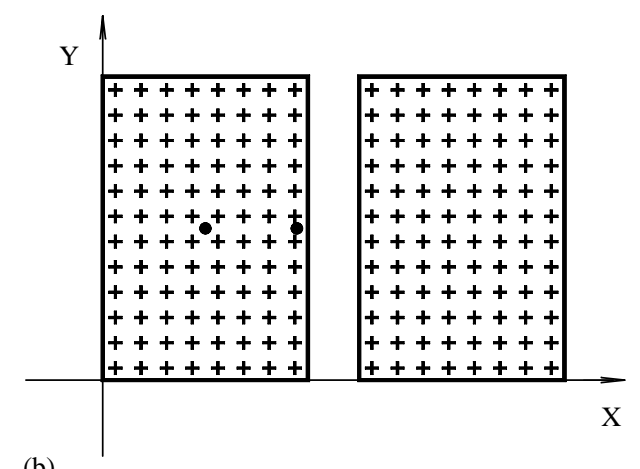

(b)

Figure 4. Numerical applications geometry: (a) position of the sources and receivers 1 and 2; (b) grid of receivers. 


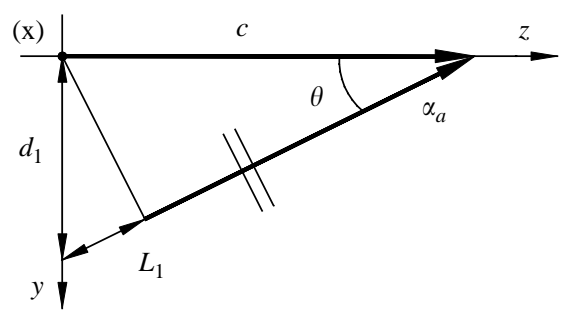

Figure 5. Apparent wave velocity.

inclination in relation to the $z$-axis are represented by $c=\infty \mathrm{m} / \mathrm{s}$, which can be understood as a pure 2-D problem, in which the source is linear. As the path inclination ranges from 90 to $0^{\circ}$, there is a lower bound value for $c$ that corresponds to the slowest wave velocities $\left(\alpha_{a}\right)$, which corresponds to waves travelling parallel to the $z$-axis. The frequency range of the computations is from 0.5 to $1000.0 \mathrm{~Hz}$ with a frequency increment of $0.5 \mathrm{~Hz}$.

A selection of results illustrating the main findings is given below. First, a separating wall $0.20 \mathrm{~m}$ thick is used to illustrate how the sound pressure level within the tunnels changes when subjected to the incidence of cylindrical waves with different spatial sinusoidal variations along the $z$-direction for the two source positions. The peaks and troughs in the Fourier amplitude spectra occur at particular frequencies and in definite frequency intervals. They are related to the eigenfrequencies of the tunnels and to the transversal vibration modes of the wall. The average sound insulation over a grid of receivers, provided by the separating wall, is then computed for different wall thicknesses and source positions.

\subsection{INCIDENCE OF CYLINDRICAL WAVES OF $k_{z}=0$}

Next, it is assumed that the $0.20 \mathrm{~m}$ thick wall is subjected to the incidence of cylindrical waves of $k_{2}=0$. This corresponds to waves arriving at the receivers with a $90^{\circ}$ inclination in relation to the $z$-axis.

Figure 6 displays responses obtained when the source is placed on the axis of the first tunnel (source 1) in the frequency range from 0.5 to $400.0 \mathrm{~Hz}$. Figure 6(a) illustrates the sound pressure level responses, in a $\mathrm{dB}$ scale, obtained at the pair of receivers $(1,2)$, placed $0.2 \mathrm{~m}$ from the separating wall at its mid-height, in the two tunnels. Receiver 1 is placed inside the tunnel, where the source is allocated, and receiver 2 is placed in the second tunnel. A source placed on the axis of the tunnel only excites modes which do not exhibit null pressure at the centre of this tunnel $[(2 q, 2 r)$ with $q=0,1, \ldots$ and $r=0,1, \ldots]$. However, part of the energy generated in the first tunnel is transmitted to the second tunnel. In the second tunnel, this energy is observed as though generated by an off-centre source placed at mid-height of both tunnels, which excites additional modes $[(q, 2 r)$ with $q=0,1, \ldots$ and $r=0,1, \ldots]$. Note that the modes $[(q, 2 r-1)$ with $q=0,1, \ldots$ and $r=1,2, \ldots]$ are not excited; this is because the source is on the horizontal symmetry axis, for which these modes registered zero pressure. The dynamic process does not stop here, since the energy in this second tunnel is also transmitted to the first tunnel, and there it can be seen as an off-centre source, exciting the additional modes. The results in Figure 6(a) agree with this interpretation: at receiver 1, the sound pressure field exhibits enhanced peaks in the vicinity of the first excited modes $\left[f_{02}=113.3 \mathrm{~Hz}, f_{20}=170.0 \mathrm{~Hz}, f_{22}=\right.$ 


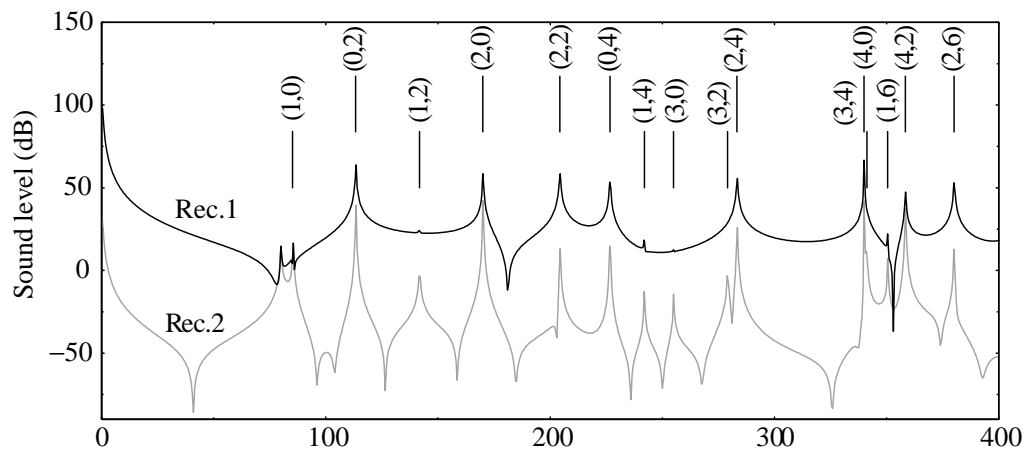

(a)

Frequency $(\mathrm{Hz})$

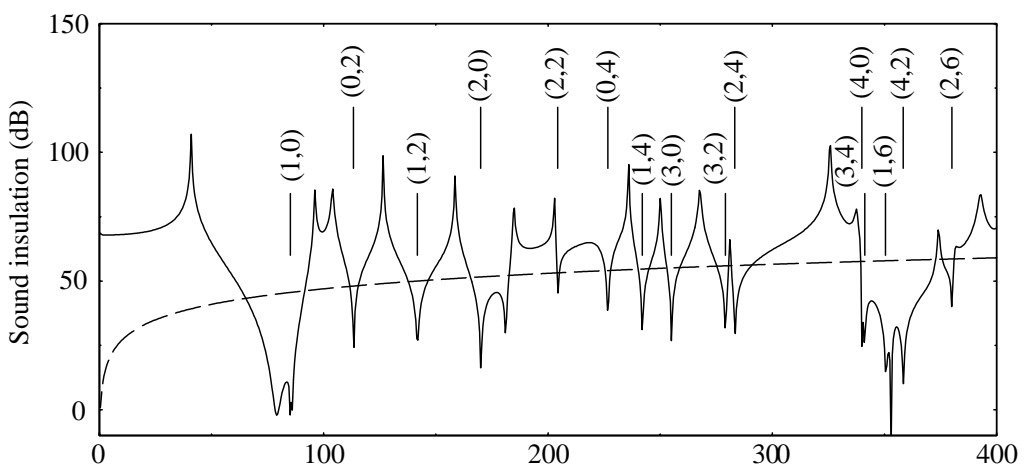

(b)

Frequency $(\mathrm{Hz})$

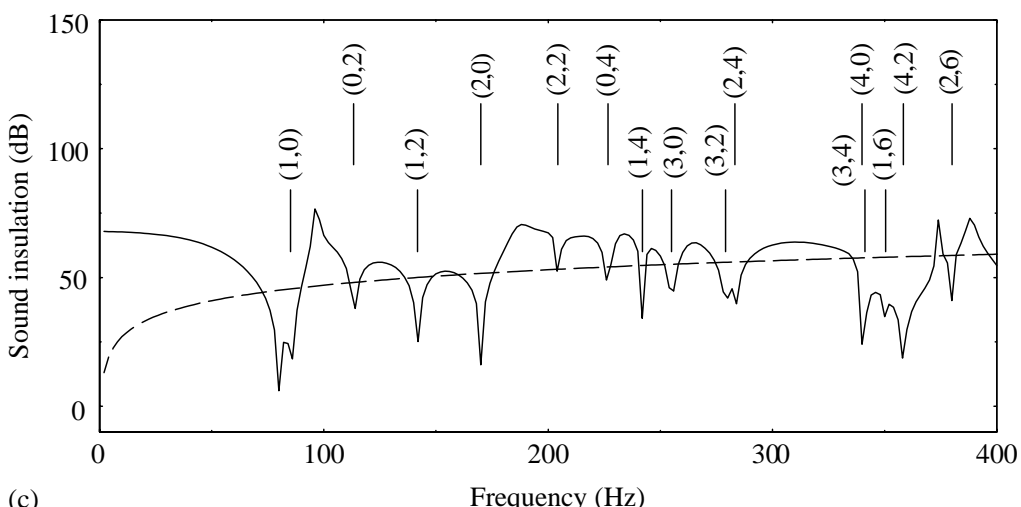

Figure 6. Responses obtained when source $1\left(k_{2}=0\right)$ is excited in the presence of a concrete wall $0 \cdot 20 \mathrm{~m}$ thick: (a) sound pressure level at receivers 1 and 2; (b) sound pressure difference between receivers 1 and $2(-)$ versus the theoretical mass law prediction $(---)$; (c) average sound insulation $(-)$ versus the theoretical mass law prediction (-- $)$.

$204.3 \mathrm{~Hz}, \quad f_{04}=226.7 \mathrm{~Hz}, \quad f_{24}=283.3 \mathrm{~Hz}, \quad f_{40}=340.0 \mathrm{~Hz}, \quad f_{42}=358.4 \mathrm{~Hz} \quad$ and $f_{26}=380 \cdot 1 \mathrm{~Hz}$ ], while additional peaks are clearly visible on the sound pressure response recorded on the second tunnel, which correspond to the excitation of the modes $\left[f_{1,0}=85.0 \mathrm{~Hz}, f_{12}=141.7 \mathrm{~Hz}, f_{14}=242.1 \mathrm{~Hz}, f_{30}=255.0 \mathrm{~Hz}, f_{32}=279.1 \mathrm{~Hz}, f_{34}=\right.$ 
$341.2 \mathrm{~Hz}$, and $f_{16}=350.5 \mathrm{~Hz}$; small peaks in the vicinity of these later modes are seen in the responses for the first tunnel, as a result of energy being transmitted from the second tunnel to the first.

Figure 6(b) plots the difference between the sound pressure levels registered at receivers 1 and 2. As expected, low insulation is observed in the vicinity of the eigenmodes of the tunnel. Figure 6(b) also includes the sound transmission loss predicted by the theoretical mass law. It can be observed that the calculated sound pressure difference between receivers 1 and 2 exhibits pronounced dips and troughs not predicted by the simplified model. These discrepancies are particularly important at lower frequencies $(<50 \mathrm{~Hz})$, for which the BEM solution indicates higher insulation values than that expected by the Mass Law.

Figure 6(c) shows the average sound insulation computed from the response obtained over a grid of 96 receivers, equally spaced $0.25 \mathrm{~m}$ along the vertical and the horizontal directions, placed in both tunnels (see Figure 4(b)). This plot is smoother than the computed pressure level difference between receivers 1 and 2 . However, it is still far from the insulation predicted by the Mass Law. The computed response appears to be highly dependent on the excited modes of the tunnels, showing poor insulation in the vicinity of the corresponding eigenfrequencies. As for receivers 1 and 2, at very low excitation frequencies, the Theoretical Mass Law predicts lower insulation than that computed by the BEM model. The results do not reveal a clear dip in insulation related to the coincidence effect. This effect would be more relevant to larger tunnels, or in cases where single panel walls are inserted in an unbounded medium. The constructive interference among the reflected fields would be weaker, leading to an enhanced contribution due to the propagation of the guided waves along the wall, for which the coupling between the solid and the fluid would be even more important.

Figure 7 displays the sound pressure level computed at receivers 1 and 2 when the separating wall is $0 \cdot 05,0 \cdot 10$ and $0 \cdot 20 \mathrm{~m}$ thick, for the frequency range from $0 \cdot 5$ to $400 \cdot 0 \mathrm{~Hz}$. The responses recorded at receiver 1 do not appear to change significantly as the thickness of the wall changes. Meanwhile, the sound pressure level registered at receiver 2 changes markedly when the wall thickness changes. As expected, the highest sound pressure level at receiver 2 is obtained when the wall is $0.05 \mathrm{~m}$ thick, while the lowest response is recorded when the wall is $0.20 \mathrm{~m}$ thick. The simplified model predicts a constant increase in sound insulation of about $6 \mathrm{~dB}$ for each doubling of mass, which does not occur with the BEM

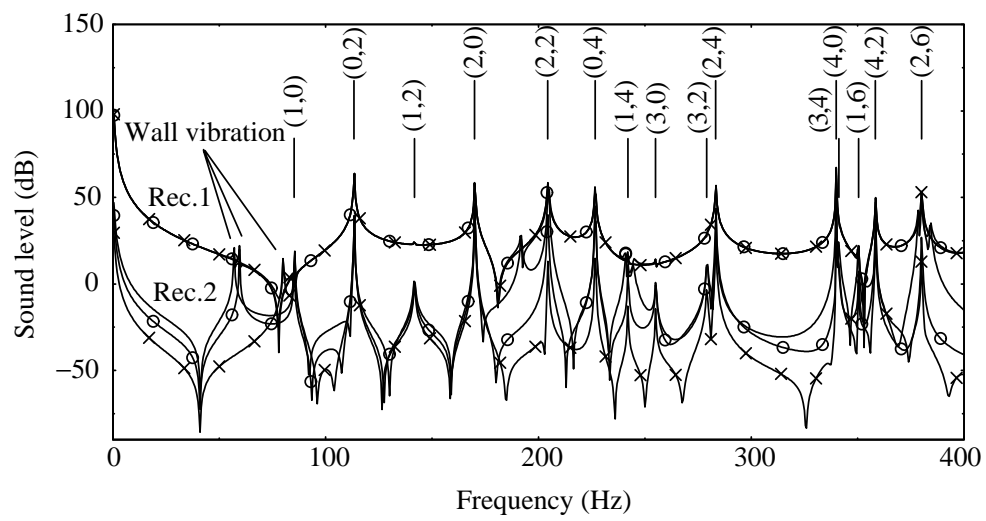

Figure 7. Sound pressure level at receivers 1 and 2 for different wall thicknesses $(-1.10 \mathrm{~m}, \bigcirc-\bigcirc 0 \cdot 10 \mathrm{~m}$ and $\times-\times 0.20 \mathrm{~m})$, when the source $1\left(k_{z}=0\right)$ is excited. 
solution. Indeed, the sound pressure difference among the various responses obtained at receiver 2 oscillates strongly throughout the frequency domain.

The peaks of the sound pressure level computed at receivers 1 and 2 are still related to the eigenmodes of the tunnels. The existence of additional enhanced sound pressure levels in the vicinity of specific frequencies not associated with the normal modes of the tunnels can also be observed. These enhanced responses are generated by the vibration of the wall, and change according to its thickness.

Figure 8 illustrates the sound pressure level obtained at receivers 1 and 2 when source 2 is excited, in the presence of a wall, $0.20 \mathrm{~m}$ thick, in the frequency range from 0.5 to $400.0 \mathrm{~Hz}$. These responses exhibit features similar to the previous ones. However, source 2 , placed away from the centre of the tunnel, excites additional eigenmodes, as the sound pressure results at receiver 1 testify.

\subsection{INCIDENCE OF CYLINDRICAL WAVES OF $k_{z} \neq 0$}

Again, a $0 \cdot 20 \mathrm{~m}$ thick wall is used to illustrate how the acoustic insulation varies when a panel is subjected to the incidence of cylindrical waves with different spatial sinusoidal variation along the axis of the tunnel to find the main features of the 3 -D effects of the acoustic insulation.

In the examples given, two apparent velocities are chosen, namely $c=400.0$ and $340.0 \mathrm{~m} / \mathrm{s}$. The apparent wave velocity $c=340.0 \mathrm{~m} / \mathrm{s}$ corresponds to waves that travel parallel to the tunnel. The waves travelling along the tunnel with an apparent wave velocity of $c=400.0 \mathrm{~m} / \mathrm{s}$ correspond to plane waves reaching the surface of the tunnels with an inclination of $58.2^{\circ}$ in relation to the normal direction of the tunnel surfaces.

The features of the responses obtained when $c=400.0 \mathrm{~m} / \mathrm{s}$ are still similar to the ones described before (see Figure 9(a)). The peaks in the sound pressure level occur at very welldefined frequency positions, which coincide with the eigenmodes of a tunnel with dimensions $3.0 \sin (31.8)=1.58 \mathrm{~m}$ and $2.0 \sin (31.8)=1.05 \mathrm{~m} \quad\left[f_{10}=161.9 \mathrm{~Hz}, f_{02}=215\right.$. $2 \mathrm{~Hz}, f_{12}=269.3 \mathrm{~Hz}, f_{20}=323.8 \mathrm{~Hz}$ and $\left.f_{22}=388.8 \mathrm{~Hz}\right]$. This happens because the journey distance $\left(L_{1}\right)$ in this domain is smaller since it corresponds to the projection of the initial vertical path $\left(d_{1}\right)$ to the inclined path, leading to a distance $L_{1}=d_{1} \sin \left[\arccos \left(\alpha_{\mathrm{a}} / \mathrm{c}\right)(\mathrm{see}\right.$ Figure 5).

The response recorded when the apparent wave velocity is $c=400.0 \mathrm{~m} / \mathrm{s}$ does not exhibit the oscillation found before (see Figure 9(b)). This behaviour was expected because

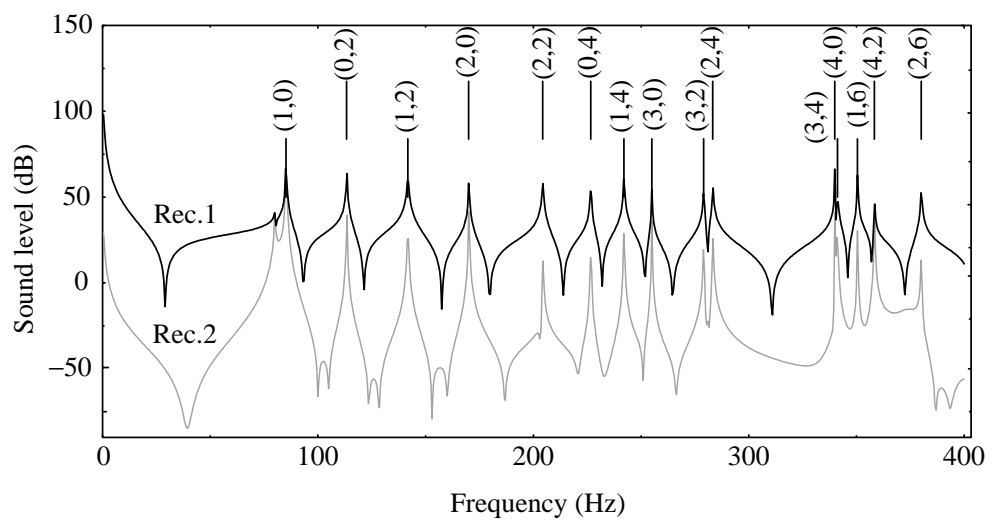

Figure 8. Computed sound pressure level at receivers 1 and 2 , when source $2\left(k_{z}=0\right)$ is excited in the presence of a $0 \cdot 20 \mathrm{~m}$ thick separating wall. 

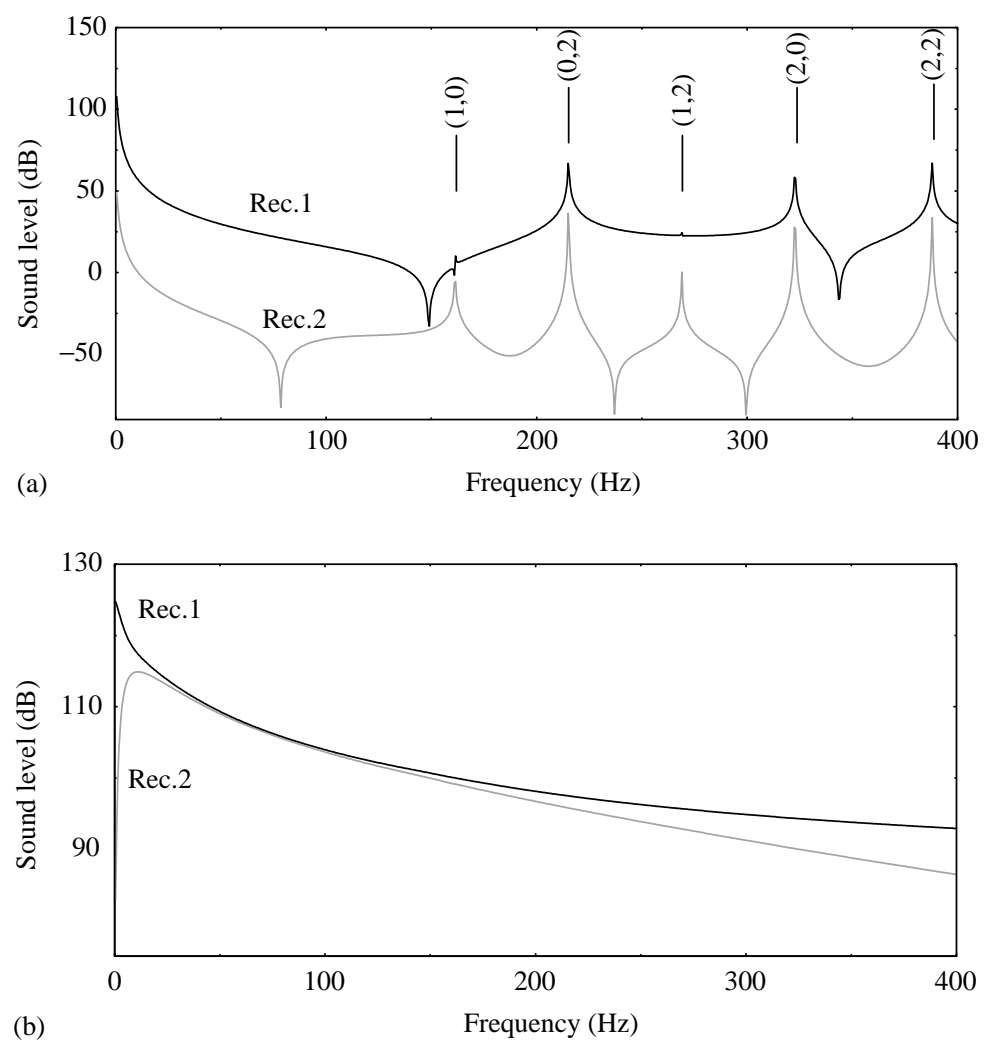

Figure 9. Sound pressure level at receivers 1 and 2 , when the $0.20 \mathrm{~m}$ thick separating wall is illuminated by a cylindrical wave with different spatial sinusoidal variations along the $z$ direction: (a) apparent wave velocity of $c=400 \cdot 0 \mathrm{~m} / \mathrm{s} ;$ (b) apparent wave velocity of $c=340.0 \mathrm{~m} / \mathrm{s}$.

the journey distance $\left(L_{1}\right)$ in this domain tends to be zero. The propagation of waves with this apparent velocity can be seen as being parallel to the $z$ direction with the wavefront being perpendicular to this direction. Thus, the reflection of these waves on the lateral walls of the tunnels is very weak, and does not lead to the constructive interference of reflected waves, registered above (see Figure 9(a)).

\subsection{AVERAGE SOUND INSULATION}

The average insulation curves computed for the various walls, with different thicknesses $(0.05,0.10$ and $0.20 \mathrm{~m})$, when source 1 is excited in the frequency range from 0.5 to $1000.0 \mathrm{~Hz}$, are now given.

Figure 10 plots the average insulation curves predicted by the BEM model when the source is linear along the $z$ direction $\left(k_{z}=0\right)$. The mass law predictions (equation (6)) are also included to illustrate the differences between the BEM model and the simplified model.

As before, at very low frequencies, the simplified model predicts insulation values lower than those obtained by the BEM model. It can also be seen that the discrepancies between the simplified insulation curves and the computed curves increase as the mass of the wall diminishes, that is, as its thickness decreases. The acoustic insulation provided by a wall of 


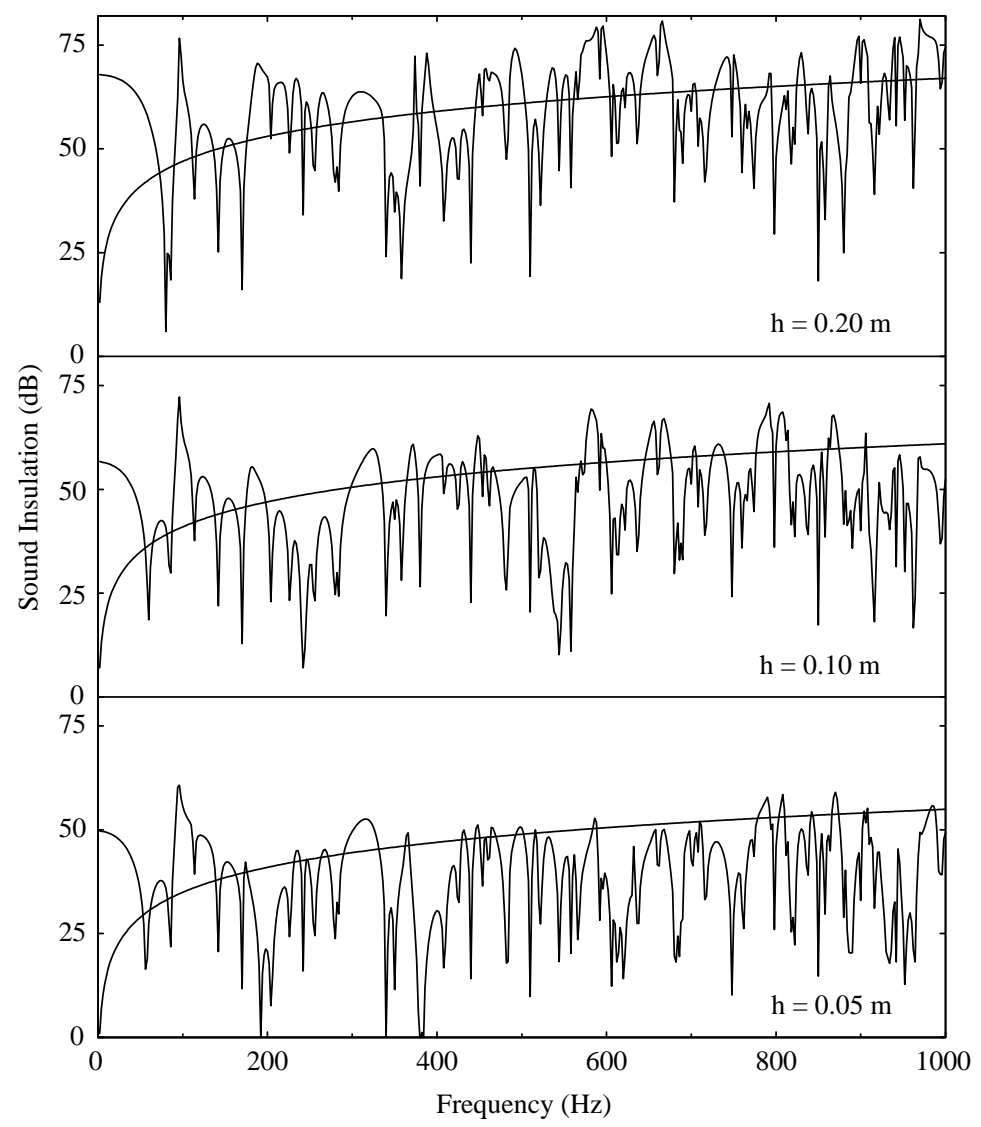

Figure 10. Average sound insulation when source $1, k_{z}=0$, is excited in the presence of separating walls of $0 \cdot 05,0 \cdot 10$ and $0 \cdot 20 \mathrm{~m}$ thicknesses.

$0.05 \mathrm{~m}$ thickness, as computed by the BEM model, is generally lower than that predicted by the simplified model, equation (6). The acoustic insulation prediction by the simplified model fits the computed insulation better when the wall is thicker $(0 \cdot 2 \mathrm{~m})$. However, the insulation computed by the BEM model is still exhibiting dips and peaks far removed from the insulation predicted by the simplified model.

Figure 11 displays the computed BEM results when the source emits cylindrical waves with an apparent wave velocity of $c=400.0 \mathrm{~m} / \mathrm{s}$. Again the insulation predicted by the simplified model is also plotted. Analysis of the results shows the sound insulation as a curve with pronounced dips. The increase in sound insulation with increasing wall mass is greater than that predicted by the simplified expression (6). The simplified model predicts an increase in sound insulation of about $6 \mathrm{~dB}$ for each doubling of mass (doubling of wall thickness), which is lower than that given by the BEM solution.

Figure 12 gives the average insulation calculated by the BEM model when the tunnel is illuminated by cylindrical waves, which exhibit an apparent wave velocity of $c=340 \cdot 0 \mathrm{~m} / \mathrm{s}$. As described above, these insulation curves do not reveal the existence of a peak/trough structure. The computed insulation responses are well below the prediction calculated by the simplified expression (6), particularly at very low frequencies, where the insulation is close to zero, regardless of wall thickness. Insulation increases for high frequencies, and the wall thickness is found to be important for the definition of insulation. 


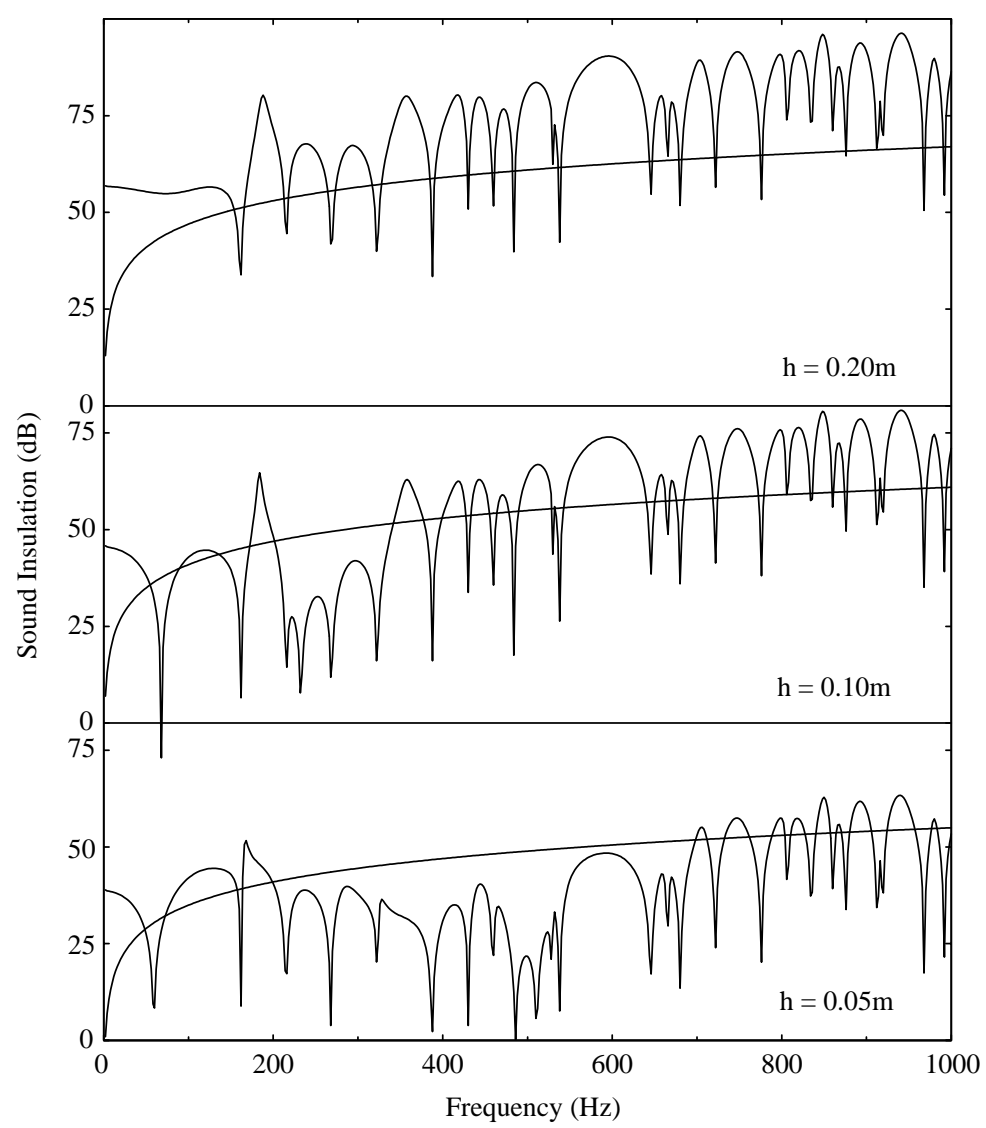

Figure 11. Average sound insulation when source 1 is excited with a spatial sinusoidal variation along the direction of $c=400.0 \mathrm{~m} / \mathrm{s}$, in the presence of separating walls of $0.05,0.10$ and $0.20 \mathrm{~m}$ thicknesses.

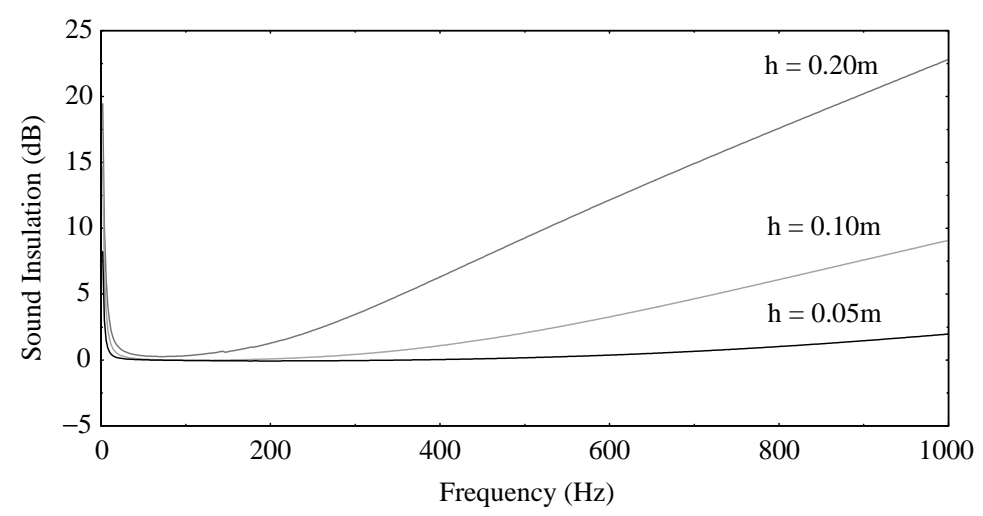

Figure 12. Average sound insulation when source 1, with a spatial sinusoidal variation along the direction of $c=340.0 \mathrm{~m} / \mathrm{s}$, in the presence of separating walls of $0.05,0.10$ and $0.20 \mathrm{~m}$ thicknesses.

\section{CONCLUSIONS}

The boundary element method (BEM) was formulated and used to compute the sound insulation provided by a wall separating two tunnels for low frequencies $(<1000 \cdot 0 \mathrm{~Hz})$. 
The model avoids the limitations in the thickness of the wall, as occurs with the Kirchhoff and Mindlin theories, and takes the coupling between the fluid (air) and the solid wall fully into account. The sound pressure results appeared to be highly dependent on the vibration modes of the tunnels. The position of the source determines the type of modes excited.

The theoretical mass law is not able to predict the oscillations in the insulation conferred by a wall, generated by the modes of the tunnel and by the bending modes of the wall, which appear to fully define its acoustic behaviour. The differences between the insulation predicted by the simplified model and that given by the BEM model are more marked as the mass of the wall diminishes, that is, as the thickness of the wall decreases. At very low frequencies, particularly, the predicted insulation is lower than the one computed by the BEM model. The simplified model should be applied with caution in the low-frequency range.

The responses obtained when the tunnels are struck by cylindrical waves with spatial sinusoidal variation along their axis display features similar to the ones observed for linear cylindrical sources. The dips of insulation in this domain appear for higher frequencies because the journey distances are shorter than the journey paths of the real domain.

The observed insulation features in this work are expected to be similar to those registered for rooms in buildings when a wall inserted between two rooms is weaker than the surrounding walls.

\section{REFERENCES}

1. J. Mathys 1993 Applied Acoustics 40, 185-200. Low frequency noise and acoustical standards.

2. B. Berglund, P. Hassmen and R. F. Soames 1996 Journal of the Acoustical Society of America 99, 2985-3002. Sources and effects of low-frequency noise.

3. W. Kropp, A. Pietrzyk and T. Kihlman 1994 Acta Acustica 2, 379-392. On the meaning of the sound reduction index at low frequencies.

4. LoRd Rayleigh 1945 The Theory of Sound. New York: Dover.

5. A. London 1950 Journal of the Acoustical Society of America 44, 270-278. Transmission of reverberant sound through double walls.

6. A. London 1949 Journal of Research and Natural Bureau of Standards 42, 605. Transmission of reverberant sound through single walls.

7. A. London 1949 Journal of Research and Natural Bureau of Standards 44, 605. Transmission of reverberant sound through double walls.

8. L. Cremer 1942 Akusticheskii Zhurnal 7, 81-102. Theorie der Shalldämmung dünner Wände bei schrägem Einfall.

9. E.C. SEWELL 1970 Journal of Sound and Vibration 12, 21-32. Transmission of reverberant sound through a single-leaf partition surrounded by an infinite rigid baffle.

10. B. H. Sharp 1978 Noise Control Engineering 11, 53-63. Prediction methods for the sound transmission of building elements.

11. L. L. BeraneK and I. L. VÉR 1992 Noise and Vibration Control Engineering, Principles and Applications. New York: Wiley.

12. J. R. Callister, A. R. George and G. E. Freeman 1999 Journal of Sound and Vibration 222 , 145-151. An empirical scheme to predict the sound transmission loss of single-thickness panels.

13. I.I. Novikov 1998 Applied Acoustics 54, 83-90. Low-frequency sound insulation of thin plates.

14. A. Osipov, P. Mees and G. Vermeir 1997 Applied Acoustics 52, 273-288. Low frequency airborne sound transmission through single partitions in buildings.

15. International Standardization Organization-ISO 140-3, 1978 Acoustics, measurement of sound insulation in buildings and of building elements, laboratory measurements of airborne sound insulation of building elements.

16. International Standardization Organization-ISO 140-10, 1989 Acoustics, measurement of sound insulation in buildings and of building elements, measurement of sound insulation of small building elements. 
17. American Society for Testing and Materials-ASTM E90, 1990 Standard test method for laboratory measurement of airborne sound transmission loss of building partitions.

18. International Standardization Organization-ISO 140-1, 1990 Acoustics, measurement of sound insulation in buildings and of building elements, requirements for laboratories.

19. L. Gagliardini, J. Roland and J.L. Guyader 1991 Journal of Sound and Vibration 145, 457-478. The use of a functional basis to calculate acoustic transmission between rooms.

20. J. Roland, M. Villenave, L. Gagliardini and D. Soubrier 1991 Contract No. 3165/1/0/078/ 87/7-BCR-B(30) CSTB. Intercomparation of measurements of noise attenuation by double glazed windows in frames.

21. D. B. Pedersen, J. Roland, G. Raabe and W. Maysenholder 2000 Acustica-Acta Acustica 86, 495-505. Measurement of the low-frequency sound insulation of building components.

22. R. J. M. Craik 1996 Sound Transmission Through Buildings Using Statistical Energy Analysis. Hampshire, England: Gower Publishing Limited.

23. E. E. Ungar 1967 Journal of Engineering and Industrial Transactions, American Society of Mechanical Engineers 87, 629-632. Statistical Energy analysis of vibrating systems.

24. M. J. Crocker and A. J. Price 1969 Journal of Sound and Vibration 9, 469-486. Sound transmission using statistical energy analysis of vibrating systems.

25. R. H. Lyon 1975 Statistical Energy Analysis of Dynamical Systems. Theory and Application. Cambridge, Massachusetts: MIT Press.

26. J. A. Steel and R. J. M. Craik 1994 Journal of Sound and Vibration 178, 553-561. Statistical energy analysis of structure-borne sound transmission by Finite Element Methods.

27. P. Hynnä, P. Klinge and J. Vuoksinen 1995 Journal of Sound and Vibration 180, 583-607. Prediction of structure-borne sound transmission in large welded ship structures using statistical energy analysis.

28. R. J. M. Craik and R. S. Smith 2000 Applied Acoustics 61, 223-245. Sound transmission through double leaf lightweight partitions. part I: airborne sound.

29. W. Kropp and E. Rebillard 1999 Acústica-Acta Acústica 85, 707-720. On the air-borne sound insulation of double wall constructions.

30. A. Osipov, P. Mees and G. Vermeir 1997 Proceedings of Inter-Noise 97 2, 759-762. Numerical simulation of airborne sound transmission at low frequencies: the influence of the room and the partition parameter.

31. S. P. S. Maluski and B. M. Gibis 2000 Journal of the Acoustical Society of America 108(4), 1741-1751. Application of a finite-element to low-frequency sound insulation in dwellings.

32. F.C. Sgard, N. Atalla and J. Nicolas 2000 Journal of the Acoustical Society of America 108, 2865-2872. A numerical model for the low frequency diffuse field sound transmission loss of double-wall sound barriers with elastic porous linings.

33. A. TAdeu and P. SAntos 2001 Journal of Soil Dynamics and Earthquake Engineering 21, 499517. 3D wave propagation in fluid-filled irregular boreholes in elastic formations.

34. M. Bouchon 1993 Geophysics 58, 475-481. A numerical simulation of the acoustic and elastic wavefields radiated by a source in a fluid-filled borehole embedded in a layered medium.

35. W. Dong, M. Bouchon and M.N. Toksöz 1995 Geophysics 60, 735-747. Borehole seismic-source radiation in layered isotropic and anisotropic media: boundary element modeling.

36. C. T. Randall 1991 Journal of the Acoustical Society of America 89, 1002-1016. Modes of noncircular fluid-filled boreholes in elastic formation.

37. J. S. Bell and D. I. Gough 1979 Earth and Planetary Science Letters 45, 475-482. Northeastsouthwest compressive stress in Alberta-evidence from oil wells.

38. Z. Zheng, K. Kemeny and N. G. W. Cook 1989 Journal of Geophysical Research 94, 7171-7182. Analysis of borehole breakouts.

39. M. Bouchon and K. Aki 1977 Bulletin of the Seismic Society of America 67, 259-277. Discrete wave-number representation of seismic source wavefields.

40. A. J. B. Tadeu, P. F. A. Santos and E. Kausel 1999 EABE-Engineering Analysis with Boundary Elements 23, 671-681. Closed-form integration of singular terms for constant, linear and quadratic boundary elements. Part I: SH wave propagation.

41. A. J. B. Tadeu, P. F. A. Santos and E. Kausel 1999 EABE-Engineering Analysis with Boundary Elements 23, 757-768. Closed-form integration of singular terms for constant, linear and auadratic boundary elements. Part II:SV-P wave propagation.

42. Y. H. PaO and C. C. Mow 1973 Diffraction of Elastic Waves and Dynamic Stress Concentrations. Santa Monica, CA, USA: Rand Corporation. 
43. K. F. Graff 1975 Wave Motion in Elastic Solids. New York: Dover Publications, INC. New York.

44. H. Kuttruff 1997 Sound in Enclosures. Encyclopedia of Acoustics, Vol. 3, pp. 1101-1115. New York: A Wiley-Interscience Publication.

45. D. OuIs 1999 Applied Acoustics 56, 1-24. Scattering by a barrier in a room.

46. R. N. S. Hammad 1988 Applied Acoustics 24, 211-228. Simulation of noise distribution in rectangular rooms by means of computer modelling techniques.

\section{APPENDIX}

\section{A. THE GEEEN'S FUNCTIONS}

\section{A.1. Solid formation}

Definitions

$$
\begin{aligned}
& \lambda, \mu \quad \text { Lamé constants } \\
& \rho \quad \text { mass density } \\
& \alpha=\sqrt{(\lambda+2 \mu) / \rho} \quad P \text { wave velocity } \\
& \beta=\sqrt{\mu / \rho} \quad S \text { wave velocity } \\
& k_{p}=\omega / \alpha, \quad k_{s}=\omega / \beta \\
& k_{\alpha}=\sqrt{k_{p}^{2}-k_{z}^{2}}, \quad k_{\beta}=\sqrt{k_{s}^{2}-k_{z}^{2}} \\
& A=\frac{1}{4 \mathrm{i} \rho \omega^{2}} \quad \text { amplitude } \\
& \gamma_{i}=\frac{\partial r}{\partial x_{i}}=\frac{x_{i}}{r}, i=1,2 \quad \text { directions cosine } \\
& H_{n \alpha}=H_{n}^{(2)}\left(k_{\alpha} r\right), H_{n \beta}=H_{n}^{(2)}\left(k_{\beta} r\right) \quad \text { Hankel functions } \\
& B_{n}=k_{\beta}^{n} H_{n \beta}-k_{\beta}^{n} H_{n \alpha} \quad B_{n} \text { functions }
\end{aligned}
$$

Green's functions for displacements

$$
\begin{gathered}
G_{x x}=A\left[k_{s}^{2} H_{0 \beta}-\frac{1}{r} B_{1}+\gamma_{x}^{2} B_{2}\right], \quad G_{y y}=A\left[k_{s}^{2} H_{0 \beta}-\frac{1}{r} B_{1}+\gamma_{x}^{2} B_{2}\right], \\
G_{z z}=A\left[k_{s}^{2} H_{0 \beta}-k_{z}^{2} B_{0}\right], \\
G_{x y}=G_{y x}=\gamma_{x} \gamma_{y} A B_{2}, \quad G_{x z}=G_{z x}=\mathrm{i} k_{z} \gamma_{x} A B_{1}, \\
G_{y z}=G_{z y}=\mathrm{i} k_{z} \gamma_{y} A B_{1} .
\end{gathered}
$$

Volumetric strain (super-index $=$ direction of load)

$$
\begin{aligned}
\varepsilon_{V o l}^{l} & =G_{x l, x}+G_{y l, y}+G_{z l, z}=A\left[\frac{\partial}{\partial x_{l}}\left(k_{s}^{2} H_{0 \beta}\right)+B_{0, x l x}+B_{0, y l y}+B_{0, z l z}\right] \\
& =A\left[\frac{\partial}{\partial x_{l}}\left(k_{s}^{2} H_{0 \beta}\right)+B_{0, x x}+B_{0, y y}+B_{0, z z}\right] \\
& =A \frac{\partial}{\partial x_{l}}\left[k_{s}^{2} H_{0 \beta}+\hat{\nabla}^{2} B_{0}\right] .
\end{aligned}
$$

$$
\text { Note : } \quad H_{0 \beta, l}=-k_{\beta} \gamma_{l} H_{l \beta}, \quad H_{0 \beta, z}=-\mathrm{i} k_{z} H_{0 \beta} \text {. }
$$


Strain components (tensor definition, not engineering)

$$
\begin{aligned}
\varepsilon_{i j}^{l} & =\frac{1}{2}\left(G_{i l, j}+G_{j l, i}\right) \\
& =\frac{1}{2} A\left(\delta_{i l} k_{s}^{2} H_{0 \beta, j}+\delta_{j l} k_{s}^{2} H_{0 \beta, i}+B_{0, i l j}+B_{0, j l i}\right) \\
& =\frac{1}{2} k_{s}^{2} A\left(\delta_{i l} H_{0 \beta, j}+\delta_{j l} H_{0 \beta, i}\right)+A B_{0, i j l} .
\end{aligned}
$$

(a) Strains for loads in the plane, $l=x, y$

$$
\begin{gathered}
\varepsilon_{V o l}^{l}=\gamma_{l} A\left(-k_{s}^{2} k_{\beta} H_{1 \beta}+k_{z}^{2} B_{1}+\frac{4}{r} B_{2}-B_{3}\right), \\
\varepsilon_{x x}^{l}=\gamma_{l} A\left(\left(\frac{2}{r} B_{2}-k_{s}^{2} k_{\beta} H_{1 \beta}\right) \delta_{x l}+\frac{1}{r} B_{2}-\gamma_{x}^{2} B_{3}\right) \\
\varepsilon_{y y}^{l}=\gamma_{l} A\left(\left(\frac{2}{r} B_{2}-k_{s}^{2} k_{\beta} H_{1 \beta}\right) \delta_{y l}+\frac{1}{r} B_{2}-\gamma_{y}^{2} B_{3}\right), \quad \varepsilon_{z z}^{l}=\gamma_{l} k_{z}^{2} A B_{1} \\
\varepsilon_{x y}^{l}=A\left(\left(\frac{1}{r} B_{2}-\frac{1}{2} k_{s}^{2} k_{\beta} H_{1 \beta}\right)\left(\delta_{x l} \gamma_{y}+\delta_{y l} \gamma_{x}\right)-\gamma_{x} \gamma_{y} \gamma_{l} B_{3}\right) \\
\varepsilon_{x z}^{l}=i k_{z} A\left(\left(\frac{1}{r} B_{1}-\frac{1}{2} k_{s}^{2} H_{0 \beta}\right) \delta_{x l}-\gamma_{x} \gamma_{l} B_{2}\right) \\
\varepsilon_{y z}^{l}=i k_{z} A\left(\left(\frac{1}{r} B_{1}-\frac{1}{2} k_{s}^{2} H_{0 \beta}\right) \delta_{y l}-\gamma_{y} \gamma_{l} B_{2}\right) .
\end{gathered}
$$

(b) Strain for axial loads, $l=z$

$$
\begin{gathered}
\varepsilon_{V o l}^{z}=i k_{z} A\left(-k_{s}^{2} H_{0 \beta}+k_{z}^{2} B_{0}+\frac{2}{r} B_{1}-B_{2}\right) \quad \varepsilon_{x x}^{z}=i k_{z} A\left(\frac{1}{r} B_{1}-\gamma_{x}^{2} B_{2}\right) \\
\varepsilon_{y y}^{z}=i k_{z} A\left(\frac{1}{r} B_{1}-\gamma_{y}^{2} B_{2}\right) \quad \varepsilon_{z z}^{z}=i k_{z} A\left(-k_{s}^{2} H_{0 \beta}+k_{z}^{2} B_{0}\right) \\
\varepsilon_{z z}^{z}=i k_{z} A\left(-k_{s}^{2} H_{0 \beta}+k_{z}^{2} B_{0}\right) \quad \varepsilon_{x y}^{z}=-i k_{z} \gamma_{x} \gamma_{y} A B_{2} \\
\varepsilon_{x z}^{z}=\gamma_{x} A\left(-\frac{1}{2} k_{s}^{2} k_{\beta} H_{1 \beta}+k_{z}^{2} B_{1}\right) \quad \varepsilon_{x z}^{z}=\gamma_{x} A\left(-\frac{1}{2} k_{s}^{2} k_{\beta} H_{1 \beta}+k_{z}^{2} B_{1}\right) \\
\varepsilon_{y z}^{z}=\gamma_{y} A\left(-\frac{1}{2} k_{s}^{2} k_{\beta} H_{1 \beta}+k_{z}^{2} B_{1}\right)
\end{gathered}
$$

(c) Stresses

$$
\tau_{i j}^{l}=\lambda \varepsilon_{V o l}^{l} \delta_{i j}+2 \mu \varepsilon_{i j}^{l}
$$

\section{A.2. Fluid formation}

\section{Definitions}

$$
\begin{aligned}
& \lambda_{a} \quad \text { Lamé constant } \\
& \rho_{a} \quad P \text { wave velocity } \\
& k_{p a}=\omega / \alpha_{a} \\
& k_{\alpha a}=\sqrt{k_{p a}^{2}-k_{z}^{2}}
\end{aligned}
$$




$$
\begin{aligned}
& A_{a}=\frac{1}{4 i} \quad \text { amplitude } \\
& \gamma_{i}=\frac{\partial r}{\partial x_{i}}=\frac{x_{i}}{r} \quad i=1,2 \quad \text { direction cosines } \\
& H_{n \alpha a}=H_{n}^{(2)}\left(k_{\alpha a} r\right) \quad \text { Hankel functions }
\end{aligned}
$$

The Green's functions for displacements

$$
G_{a x}=-A_{a} k_{\alpha a} H_{1 \alpha a} \gamma_{x}, \quad G_{a y}=-A_{a} k_{\alpha a} H_{1 \alpha a} \gamma_{y} .
$$

Stresses

$$
H_{a 1}=A_{a} \lambda_{a} H_{0 \alpha a}\left(-\omega^{2} / \alpha_{a}^{2}\right)
$$

